\title{
Analyzing the Impact of Diverse PAPR Reduction Techniques on BER Performance of OFDM System
}

\author{
Shiwani Sandal and Lavish Kansal \\ School of Electronics and Electrical Engineering, \\ Lovely Professional University, Punjab, India \\ shiwanisandal@gmail.com,lavish.15911@lpu.co.in
}

\begin{abstract}
Orthogonal frequency division multiplexing (OFDM) is mostly considered because of its highly efficient bandwidth capability, high data rate and ability to prevent multipath fading. With the progression in age and its developing requests, there has been fast development in the field of communications. In any case the vast peak - to - average power ratio (PAPR) of these signal and high bit error rate (BER) have some undesirable impacts on the framework. In this work, diverse transforms, clipping and some hybrid techniques are proposed to reduce the PAPR of the signal and enhance the BER performance. Simulation results show that there is a considerable reduction in PAPR of the signal by using diverse transforms and clipping technique. BER performance is also better than the conventional OFDM systems in many simulation frameworks.
\end{abstract}

Keywords: OFDM, PAPR, BER, Clipping, Transforms, Hybrid reduction technique

\section{Introduction}

Lately, the necessity of multimedia wireless data services has grown extravagantly which bring the communication in the era of 4th generation wireless communication system. In this era where some of users are more with restricted bandwidth, the need for modern digital wireless communication is adopted because it provides better spectral efficiency with efficient bandwidth and also robust to multipath channel environment which is known as a multi-carrier communication system. This type of communication system provides an ease of high-speed data rate at cheap cost for many users with high reliability. The main difference between single carrier system and multi-carrier system is that in single carrier system, single carrier occupies entire communication bandwidth but in multicarrier available bandwidth is distributed among many sub-carriers so that each subcarrier carries the useful amount of bandwidth according to its need as compare to the whole bandwidth as in case of single carrier system [1]. These enormous features of the multicarrier system draw our attention to study Orthogonal Frequency Division Multiplexing (OFDM). OFDM is the base for all $4 \mathrm{G}$ wireless communication systems because it has the large capacity (number of subcarriers), high data rate (excess of $100 \mathrm{Mbps}$ ), and efficient use of bandwidth, receiver simplicity, and ubiquitous coverage with high mobility. OFDM is getting so well known since it is less presented to multipath impacts that make it the centre method for the future standard frameworks, beside the high efficiency of its spectrum. The principle disadvantage of OFDM is the peak to average power proportion (PAPR) execution, which ascends so high because of nonlinear effects of amplifiers. The high PAPR lessens the efficiency of the output power amplifier. There are a few procedures, to lessen the PAPR, which are isolated into various classes: reduction techniques, precoding techniques, reduction with transform, block coding technique, companding technique and so on. In this paper, we will use clipping reduction technique and combine with different

Received (November 19, 2017), Review Result (February 5, 2018), Accepted (February 15, 2018) 
transforms and precoding technique which work as a hybrid technique [2]. The paper is composed as take after: In Section II and III a short meaning of the OFDM and the PAPR to express the fundamental ideas. At that point, a brief clarification and portrayal of the system in area IV. In segment $\mathrm{V}$ a recreation result is put with amplified points of interest. At long last in area VI, we finish up this paper with some of our perceptions.

\section{System Description}

The basic principle of OFDM system uses the concept of frequency division multiplexing i.e., to split the wide frequency band into small frequency bands and carries data onto each of these subcarriers and transmitted in the simultaneous manner which is known as multiplexing. Data is transmitted parallelly with a longer period which removes ISI by using guard interval or cyclic prefix [3]. Also, it follows the orthogonality principle so that each subcarrier is orthogonal to each other (i.e., there cross-correlation is 0 and autocorrelation is 1) which is very helpful to transmit the data on subcarriers in a very efficient manner.

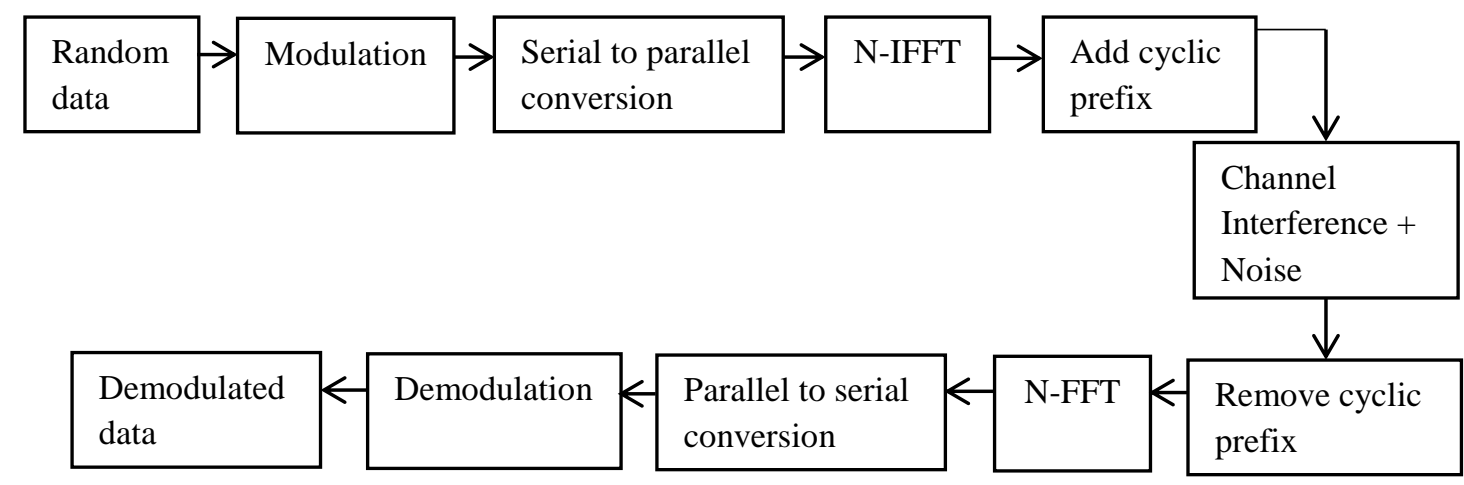

Figure 1. Basic Block Diagram of OFDM System

An OFDM system having $\mathrm{N}$ sub-carriers $\mathrm{x}(\mathrm{t})$, where $0<\mathrm{t}<\mathrm{T}$ consist of $\mathrm{N}$ complex baseband data i.e., $\mathrm{x} 0, \mathrm{x} 1, \mathrm{x} 2, \ldots \ldots \ldots \mathrm{x}_{\mathrm{n}-1}$ carried on to $\mathrm{N}$ subcarriers and transmitted on a channel simultaneously[4]. These subcarriers are orthogonal to each other with a constant spacing provided by the guard band.

The signal $\mathrm{x}(\mathrm{t})$ is defined by:

$$
x(t)=\frac{1}{\sqrt{ } N} \sum_{K=0}^{N-1} X_{\mathrm{k}} e^{j 2 \pi \Delta f t}, \quad 0 \leq \mathrm{t} \leq \mathrm{T}
$$

Though OFDM has many advantages like better spectral efficiency, high data rate up to $100 \mathrm{Mbps}$ and so on but the major drawback occur in a system are high Peak to average power ratio and high bit error rate which make this system inefficient [5].

\section{$>$ Peak to Average Power Ratio (PAPR)}

High Peak-to-Average Power Ratio has been perceived as one of the major functional issues including OFDM balance. High PAPR happens when the signal is changed over into time domain through IFFT the subsequent signal is sum the of $n$ number of the subcarrier and the peak of resulting signal gets to be $\mathrm{n}$ times higher than the normal averaged signal. Power amplifier works in resulting intermodulation distortion in the transmitted signal. This problem can be removed by making the power amplifier to work in the linear region [6]. Such power reduction results in bad performance or inefficient performance of the OFDM system.

On account of OFDM, these subcarriers are orthogonal and with consistent inter carrier spacing $\Delta \mathrm{f}=1 / \mathrm{T}$, where $\mathrm{T}$ is the OFDM symbol duration [7]. The complex baseband representation of a multicarrier signal comprising of $\mathrm{K}$ subcarriers is given 


$$
x(t)=\sum_{n=0}^{N-1} X_{\mathrm{k}} e^{j 2 \pi f t} \quad 0 \leq t \leq N T
$$

PAPR is defined as the peak signal power versus the average signal power. The PAPR of a signal is expressed by the following formula

$$
P A P R_{d b}=10 \log \left(\frac{\max x(t) x *(t)}{E x(t) x *(t)}\right)
$$

Where ()* represents the conjugate operator.

\section{Bit Error Rate (BER)}

BER is the ratio of erroneous bits to the total number of bits that have been transmitted over a given period [9]. It is expressed as 10 to the negative power. It is a major problem in OFDM system because as we reduced the PAPR, BER further goes on increasing at a drastic rate.

$$
B E R=\frac{\text { Number of errors }}{\text { Total number of bits sent }}
$$

Causes of BER are interference, increase transmitted power, lower order modulation, reduce bandwidth.

\section{PAPR Reduction Techniques}

\section{(a) Clipping:}

This technique comes under signal distortion technique though it is simple to implement, it causes in out of band radiation and in-band distortion which causes interference of the neighbouring signal [10]. Here we clipped the modulated data depending upon the user who set up the threshold value of the clipping. Up to that value, clipping is done.

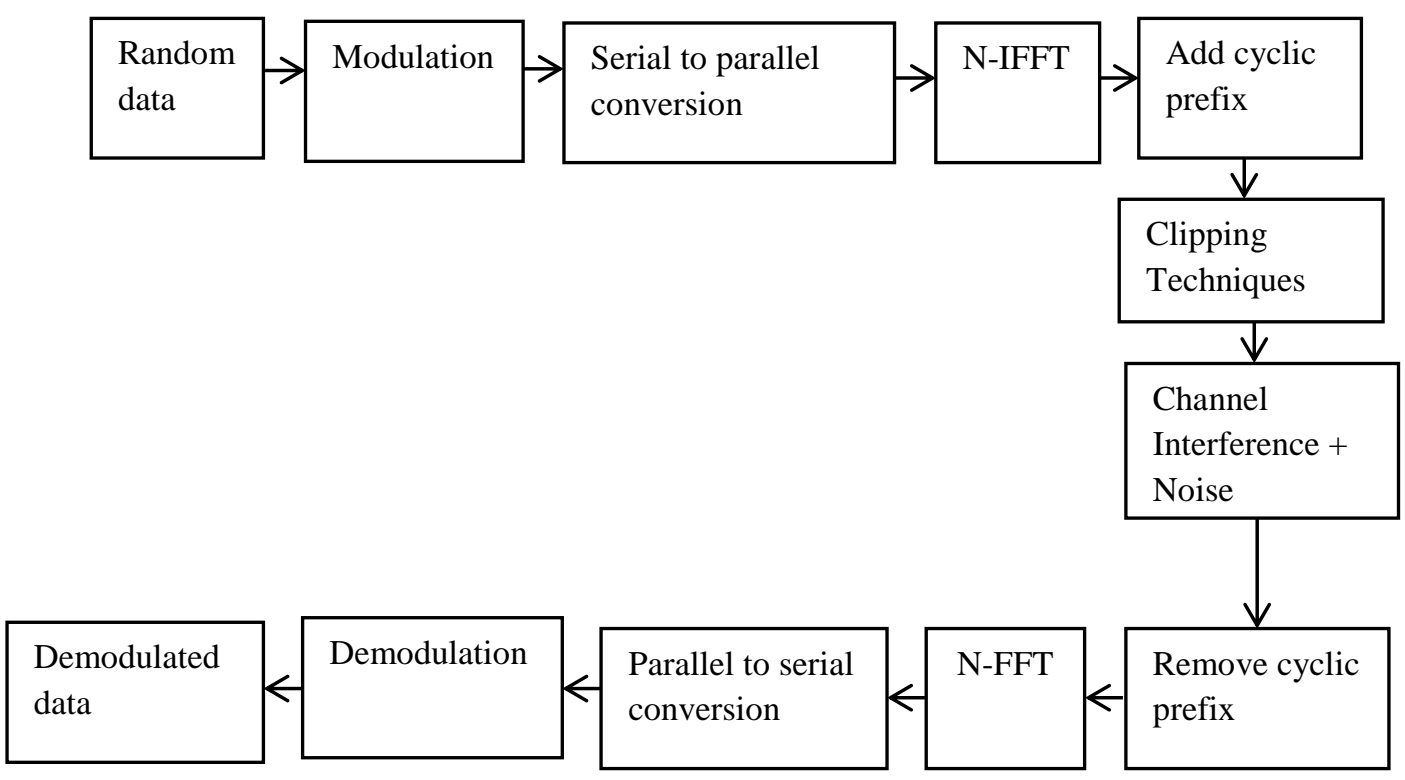

Figure 2. Reduction with Clipping Technique in OFDM System

\section{(b) Reduction with Transform:}

In this technique, we replace the IFFT (inverse fast Fourier transform) other diverse transforms [11]. Different type of transforms is available are: DWT (discrete wavelet transform), DHT (discrete Hartley transform), DST (discrete sine transform), DCT (discrete cosine transform) and so on. DFT causes leakage effect which is overcome by using DCT and DST, but these transforms functions are not flexible, so DWT is mostly used for better flexibility and getting the good efficiency in a system. 


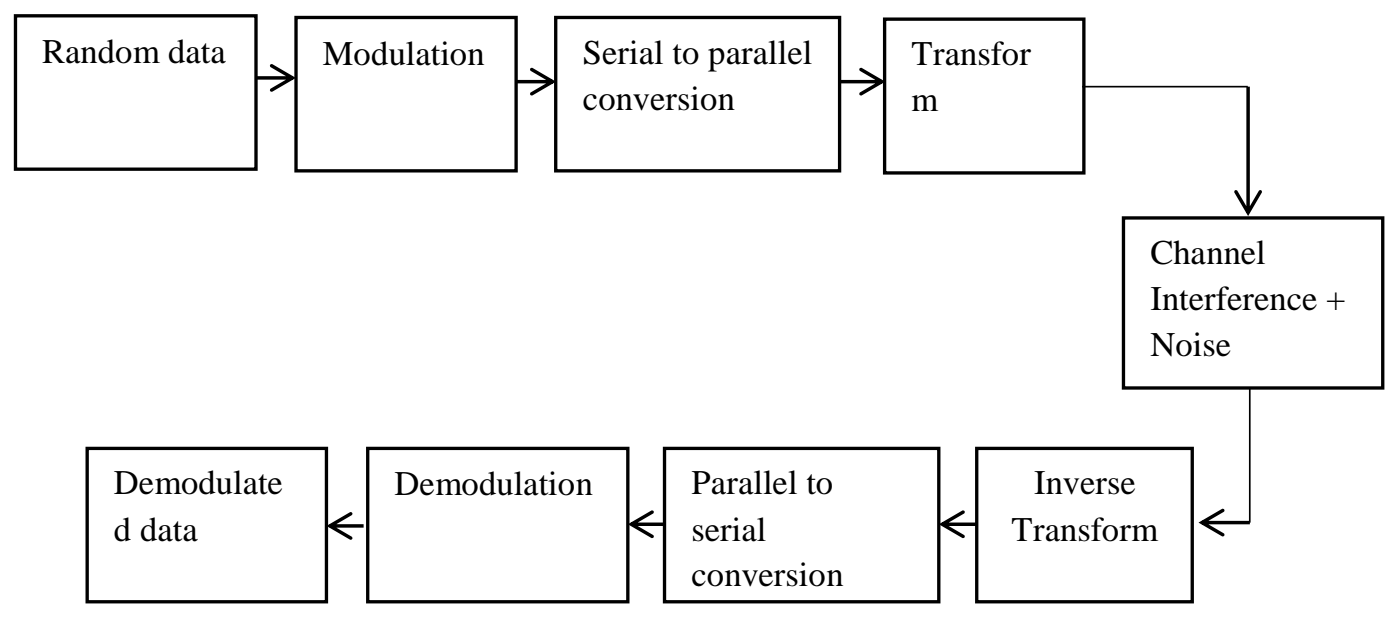

Figure 3. Reduction with Transform in OFDM System

\section{- DCT (Discrete Cosine Transform)}

A discrete cosine transform (DCT) expresses a finite sequence of data points regarding a sum of cosine functions oscillating at different frequencies.

$$
\begin{aligned}
& {\left[\frac{1}{\sqrt{ }}, \quad \mathrm{i}=0,0 \leq \mathrm{j} \leq \mathrm{M}-1\right.} \\
& \left\lfloor\sqrt{2} / \sqrt{ } M \cos \frac{(2 j+1) i \pi}{2 M} \quad 0 \leq \mathrm{i}, \mathrm{J} \leq M-1,\right.
\end{aligned}
$$

Where $\mathrm{i}, \mathrm{j}$ are row and columns respectively.

\section{- DST (Discrete Sine Transform)}

It is equivalent to the imaginary parts of a DFT of roughly twice the length, operating on real data with odd symmetry (since the Fourier transform of a real and odd function is imaginary and odd), where in some variants the input and/or output data are shifted by half a sample [12].

$$
\mathrm{Y}(\mathrm{k})=\sum_{n-1}^{N} x(n) \sin \pi \frac{k n}{N+1} \mathrm{k}=1, \ldots \ldots \ldots \mathrm{N}
$$

$\mathrm{x}$ is input matrix, $\mathrm{y}$ is output.

\section{(c) Precoding Technique:}

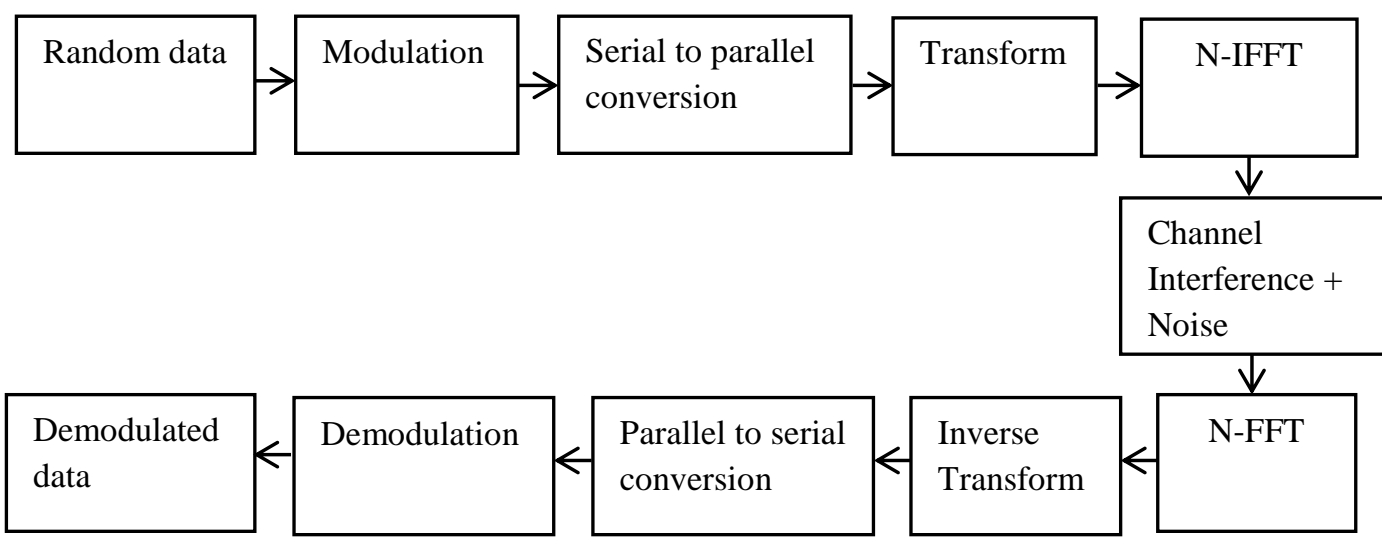

Figure 4. Precoding Technique in OFDM System 
In this technique, we are using the transform before the IFFT block at the transmitter side and after the FFT inverse of that transform is used at the receiver side. Discrete-Cosine transform matrix (DCTM) precoding method is good for improving PAPR as well as BER. In this technique, constellation symbols are precoded with linear independent precoder, which diminishes the autocorrelation relationship of the IFFT input sequence and disperses the information among subcarriers. Another method Hadamard precoding, Zad-off chu precoding method is used but as compared to DCTM precoding they are more complex[12]. We used different transform like DHT(discrete Hartley transform), DWT(discrete wavelet transform), DFT(discrete Fourier transform), DST(discrete sine transform), DCT(discrete cosine transform) and another transform in combination of IFFT OFDM block which produces precoded scheme and we check the PAPR and BER in each case and check which precoding technique is best.

\section{(d) Hybrid Technique:}

The combination of DCT precoding with clipping can lower the PAPR in very efficient manner. Firstly, by DCT data is transformed into new modified form. Secondly, the proposed scheme utilizes the clipping method. In this scheme first, the transmitted data are transformed with the help of DCT precoding matrix before IFFT operation, which reduces the PAPR. After that clipping is done.

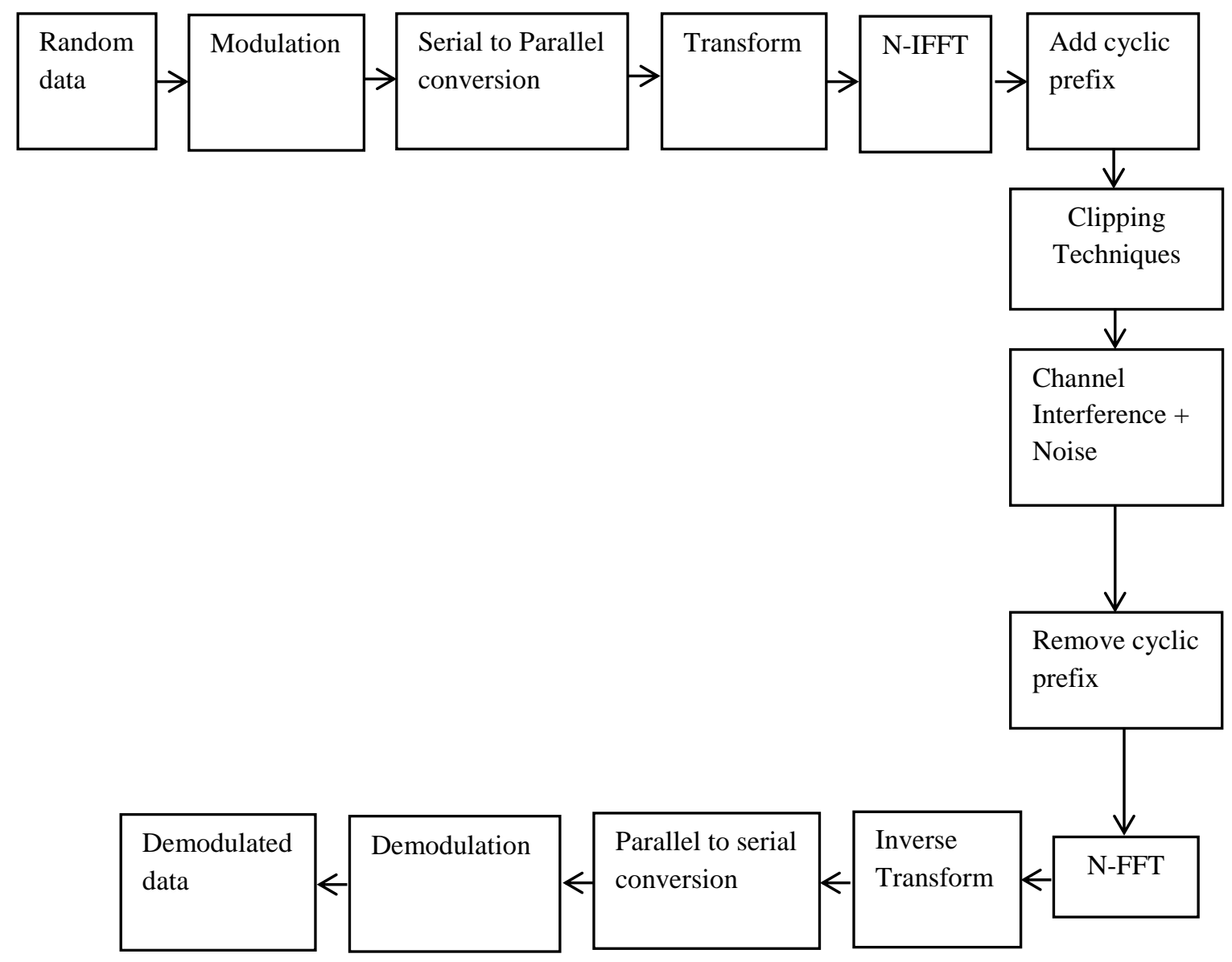

Figure 5. Reduction with Precoding Transform in OFDM System

\section{Results Discussion}

PAPR and BER performance are performed in following scenarios: 


\section{(a) Analysis of OFDM System}

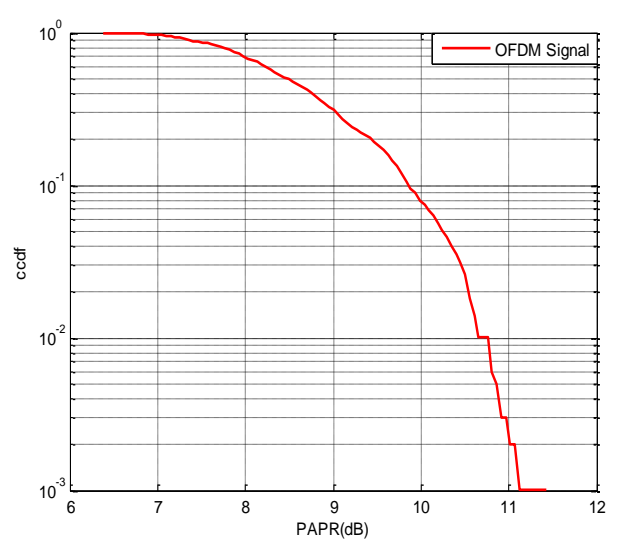

Figure 6 (a). PAPR of OFDM Signal

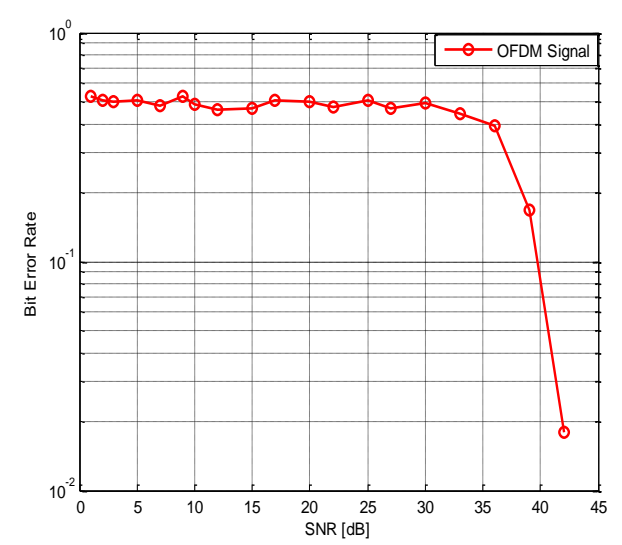

Figure 6 (b). SNR versus BER of OFDM Signal

Figure 6 (a) and (b) shows the PAPR and BER of simple OFDM signal which is $15 \mathrm{~dB}$ and $45 \mathrm{~dB}$ respectively which is quite high. These results are for simple OFDM signal i.e., without any reduction technique and precoding technique.

\section{(b) Analysis of OFDM System with Clipping Reduction Technique}

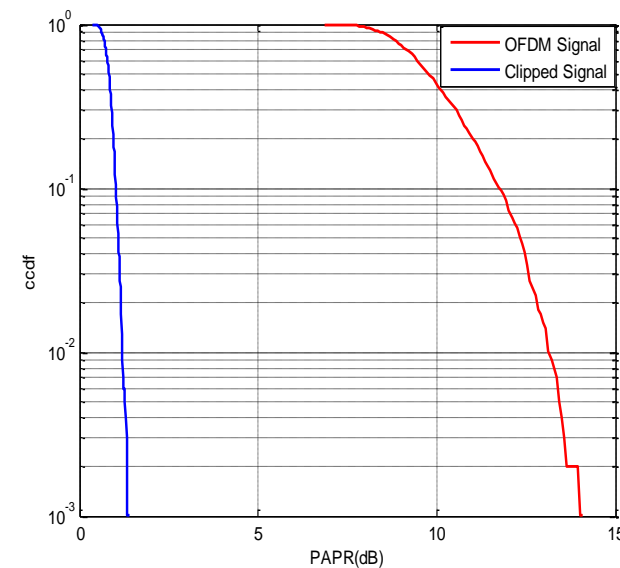

Figure 7 (a). PAPR of OFDM Signal with Clipped Signal

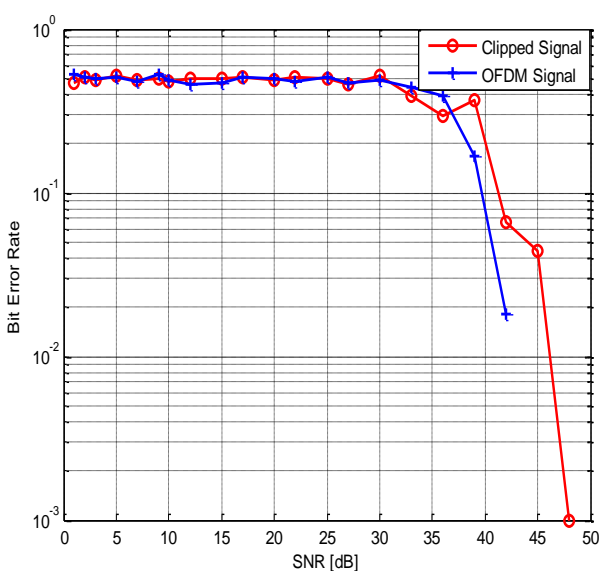

Figure 7 (b). SNR versus BER of OFDM Signal with Clipped Signal

Figure 7 (a) and (b) shows the PAPR and BER of OFDM signal with reduction technique clipping are used and comparison analysis made between the OFDM signal and Clipped signal. PAPR of the OFDM system is $14 \mathrm{~dB}$ approximately and that of Clipped signal it is $2 \mathrm{~dB}$ which is quite less. This same analysis is made in the case of SNR verses BER analysis in which BER of OFDM signal and Clipped signal are approximately same.

\section{(c) Analysis of OFDM with Transforms}

In Figure 8(a) comparison of PAPR is done between Transforms and conventional OFDM. PAPR of conventional OFDM, DCT transform, DWT transform is $12 \mathrm{~dB}, 14 \mathrm{~dB}$, $11 \mathrm{~dB}$ respectively which shows that DWT transform has lower PAPR as compared to all. In Figure 8(b) SNR verses BER analysis done between the transforms and conventional 
OFDM. BER of conventional OFDM, DCT transform and DWT transform are 48dB, 10dB, $12 \mathrm{~dB}$ approximately.

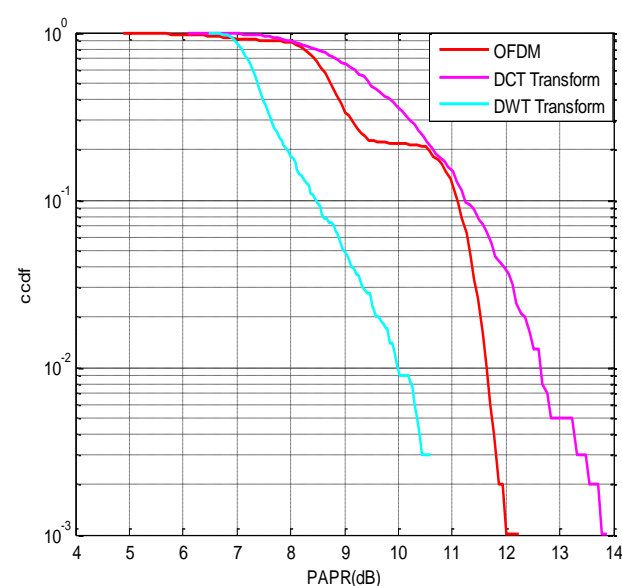

Figure 8 (a). PAPR of DCT and DWT Transforms with OFDM Signal

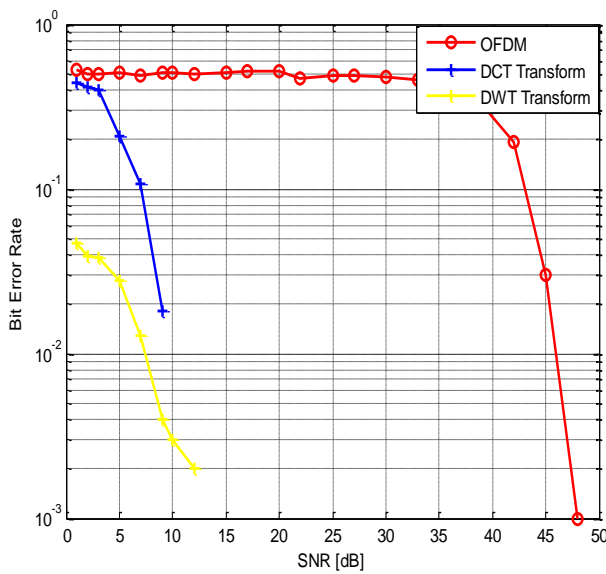

Figure 8 (b). SNR versus BER of DCT and DWT Transforms with OFDM Signal

(d) Analysis of OFDM System with Precoding

In Figure 9(a) PAPR comparison is done between different precoding schemes with conventional OFDM signal. PAPR of OFDM, DCT, DST and DWT Precoding are 12dB, $13.5 \mathrm{~dB}, 13.5 \mathrm{~dB}, 11.2 \mathrm{~dB}$ respectively. In Figure 9(b) same analysis is done for SNR verses BER for conventional OFDM, DCT, DST, DWT Precoding which is $49 \mathrm{~dB}, 12 \mathrm{~dB}, 16 \mathrm{~dB}$, $45 \mathrm{~dB}$ respectively.

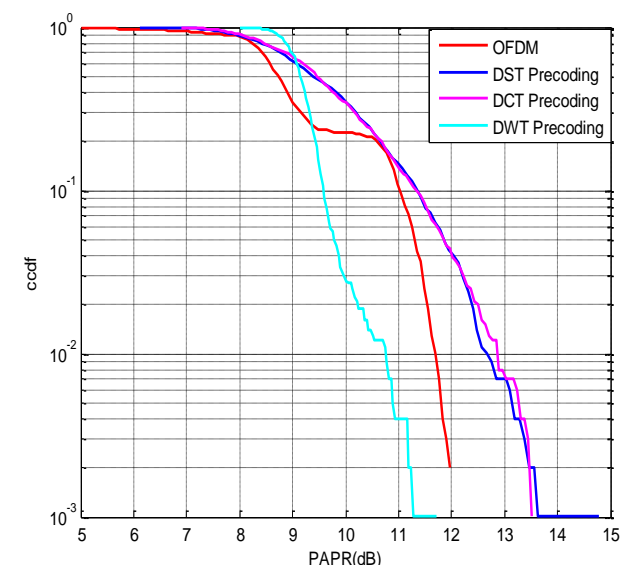

Figure 9 (a). PAPR Comparison of DST, DWT, DCT Precoding with OFDM Signal

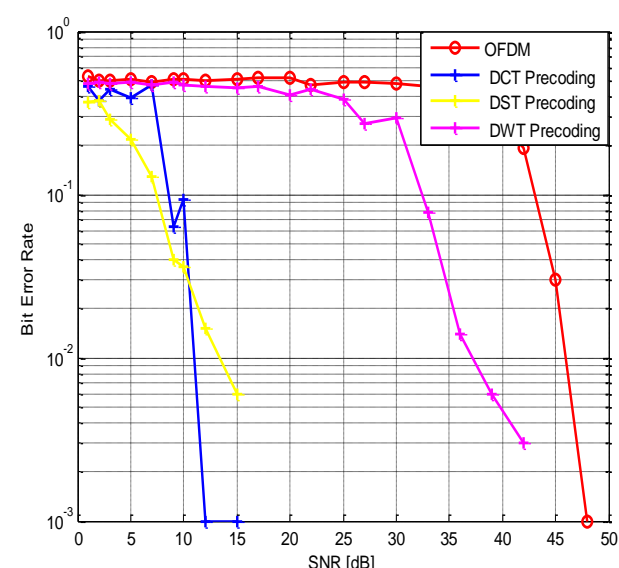

Figure 9 (b). SNR versus BER Comparison of DCT, DST and DWT Precoding with OFDM Signal

(e) Analysis of OFDM System with Hybrid Technique

> Precoded with Reduction Technique Clipping

In Figure 10(a) PAPR comparison is done between OFDM signal with hybrid Clipping and different precoding schemes. PAPR of OFDM signal is $11 \mathrm{~dB}$ where PAPR of hybrid 
schemes i.e., DST, DWT, DCT precoding with clipping are $1 \mathrm{~dB}, 4.2 \mathrm{~dB}, 4.3 \mathrm{~dB}$ approximately. So by using clipping with precoded signal PAPR reduced at a very satisfactory level. The same analysis is done in Figure 10(b) for SNR verses BER. For hybrid schemes i.e., DST, DWT, DCT precoding with clipping reduction technique are $50 \mathrm{~dB}, 40 \mathrm{~dB}$ and $50 \mathrm{~dB}$ approximately which is very much high. At one side we get good performance of PAPR, but on the other side, high BER is there. So the trade-off is there between PAPR and BER.

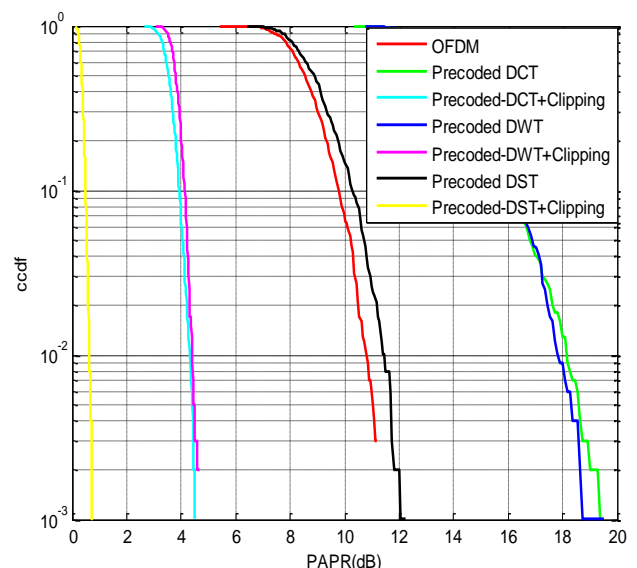

Figure 10 (a). PAPR of OFDM Signal with Hybrid Clipping and DST, DWT, DCT Precoding

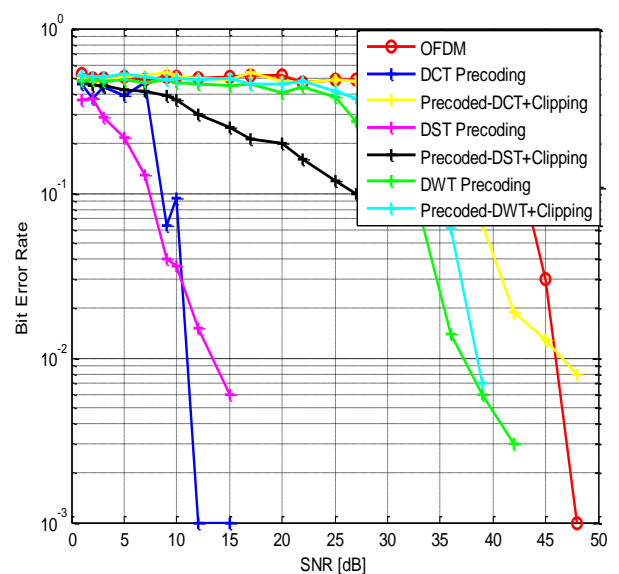

Figure 10 (b). SNR versus PAPR of OFDM Signal with Hybrid Clipping and DST, DCT, DWT Precoding

$>$ Analysis of OFDM system with Transform with Reduction Technique Clipping

In Figure 11(a) PAPR comparison is done between OFDM signal with hybrid Clipping and different Transforms schemes. PAPR of OFDM signal is $11 \mathrm{~dB}$ where PAPR of hybrid schemes i.e. DWT, DCT Transforms with clipping are $2 \mathrm{~dB}, 1 \mathrm{~dB}$. So by using clipping with transformed signal PAPR reduced at a very satisfactory level. The same analysis is done in Figure 11(b) for SNR verses BER. For hybrid schemes i.e., DWT, DCT Transforms with clipping reduction technique are $13 \mathrm{~dB}, 35 \mathrm{~dB}$ approximately which is very much high.

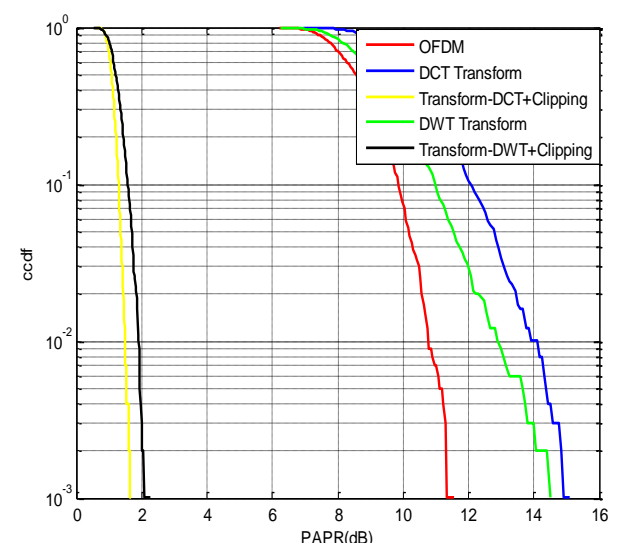

Figure 11 (a): PAPR of OFDM Signal with Hybrid Clipping and DCT, DWT Transforms

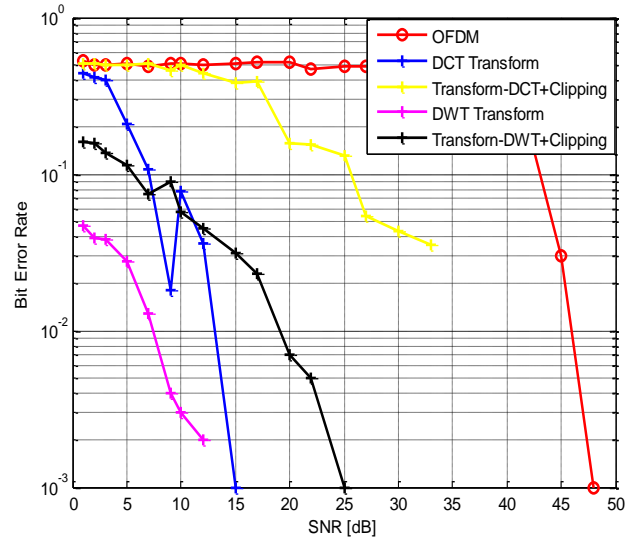

Figure 11 (b): SNR versus BER of OFDM Signal with Hybrid Clipping and DCT, DWT Transforms 


\section{Conclusion}

OFDM is a very much attractive scheme for multicarrier transmission as it is known as MCM and has better spectral efficiency, simple deployment of receiver section and highspeed data transmission up to $100 \mathrm{mbps}$ over a communication channel. In this paper, the simulations have been carried out for OFDM systems employing diverse transforms along with clipping technique. Every scheme has pros and cons, some of the simulations showing a significant decrease in PAPR, on the other hand, the BER performance for the same has been deteriorated. Similarly, in some schemes BER performance has been enhanced significantly but at the expense of elevated PAPR value. However, some schemes do show a superior BER and PAPR reduction performance in comparison to conventional OFDM system.

\section{References}

[1] Y. Wang, "PAPR reduction of an OFDM signal by use of PTS with low computational complexity", IEEE Transaction on Broadcasting, vol. 52, no. 1, (2006), pp. 83-86.

[2] T. Jiang "PAPR reduction of OFDM signals using partial transmit sequences and Reed- Muller codes," IEEE Communications Letters, vol. 11, no. 6, (2007), pp. 528-530.

[3] X. Li and L. J. Cimini, "Effects of clipping and filtering on the performance of OFDM", IEEE Communication Letters, vol. 2, no. 5, (1998), pp. 131-133.

[4] S. H. Han and J. H. Lee, "An overview of peak-to-average power ratio reduction techniques for multicarrier transmission", IEEE Wireless Communication. Magazine, vol. 12, no. 2, (2005), pp. 56-65.

[5] I. Hosseini, M. J. Omidi, K. Kasiri, A. Sadri and P. G. Gulak, "PAPR Reduction in OFDM Systems Using Polynomial-Based Compressing and Iterative Expanding", IEEE International Conference on Acoustics Speech and Signal Processing Proceedings, vol. 4, (2006), pp. 4-6.

[6] S. Y. L. Goff, B. K. Khoo, C. C. Tsimenidis and B. S. Sharif, "A Novel Selected Mapping Technique for PAPR Reduction in OFDM Systems", IEEE Transactions on Communication, vol. 56, no. 11, (2008), pp. 1467-1501.

[7] L. Renze, J. Longjiang, L. Lang, L. Jie and Z. Weile, "Reducing the Peak-to-Average Power Ratio of OFDM system with low complexity", Journal of Electronics (China), vol. 23, no. 1, (2006), pp. 26-28.

[8] M. M. Hasan, "PAPR Reduction in OFDM Systems Based on Autoregressive Filtering", Circuits, Systems and Signal Processing, vol. 33, no. 5, (2014), pp. 1637-1654.

[9] C. Duanmu \& H. Chen, "Reduction of the PAPR in OFDM Systems by Intelligently Applying Both PTS and SLM Algorithms", Wireless Personal Communications, vol. 74, no. 2, (2014), pp. 849-863.

[10] B. M. Lee, Y. Kim and R. J. P. de Figueiredo, "Performance Analysis of the Clipping Scheme with SLM Technique for PAPR Reduction of OFDM Signals in Fading Channels", Wireless Personal Communications, vol. 63, no. 2, (2012), pp. 331-344.

[11] A. Chakrapani and V. Palanisamy, "A Novel Clipping and Filtering Algorithm Based on Noise Cancellation for PAPR Reduction in OFDM Systems", Proceedings of the National Academy of Sciences, India Section A: Physical Sciences, vol. 84, no. 3, (2014), pp. 467-472.

[12] I. Baig and V. Jeoti, "A new DCT matrix precoding based RI-OFDMA uplink system for PAPR reduction”, 4th International Conference on Intelligent and Advanced Systems, (2012), pp. 680-684. 
International Journal of Control and Automation

Vol. 11, No. 5 (2018) 